\title{
RECONOCIMIENTO DE PALABRAS: UN EXPERIMENTO A PARTIR DE LA TAREA DE DECISIÓN LÉXICA
}

\author{
Anita Arrieta Espinoza
}

\begin{abstract}
RESUMEN
En este artículo se evalúa la posible relación entre la velocidad a la cual se procesan las palabras morfológicamente complejas y sus respectivos grados de transparencia semántica y de afijación, con el fin de inferir efectos explicables por los modelos de reconocimiento de palabras habladas polimorfémicas.
\end{abstract}

\begin{abstract}
This article evaluates the possible relationship between the speed at which morphologically complex words are processed and their respective grades of semantic transparency and of affixing with the intent to infer explainable effects through the method of understanding spoken polymorphemic words.
\end{abstract}

En una etapa anterior de esta investigación (Arrieta 2003), se obtuvieron, con la aplicación de cuestionarios escritos y auditivos, los juicios correspondientes al grado de transparencia semántica ${ }^{1}$ y al grado de prefijación y de sufijación ${ }^{2}$ de los estímulos seleccionados.

En esta segunda etapa, nos proponemos evaluar, mediante el empleo de una tarea ${ }^{3}$ de decisión léxica ${ }^{4}$, si las características de los ítemes (ver punto 1.2.5.2) tienen influencia sobre el tiempo de reacción ${ }^{5}$ que toman los sujetos implicados en esta investigación para reconocer las palabras habladas polimorfémicas. Evaluaremos si existe relación entre la velocidad a la cual las palabras morfológicamente complejas son tratadas y sus respectivos grados de transparencia semántica y de afijación (prefijación y sufijación), obtenidos por medio de la aplicación de cuestionarios, como se detalló en un artículo anterior, ya citado.

\section{Influencia de la morfología en el procesamiento de palabras habladas morfológicamente complejas}

Una de las principales inquietudes que se plantean en el campo de la psicolingüística a propósito de la morfología es determinar si las palabras morfológicamente complejas se someten, o no lo hacen, a un análisis morfológico durante su tratamiento, dentro del proceso de reconocimiento. Al respecto, recordemos que las investigaciones de Marslen-Wilson et al. 
(1994) y de Meunier (1997) muestran la influencia de información morfológica sobre el reconocimiento de palabras morfológicamente complejas en la modalidad auditiva (véase Arrieta 2003).

\section{Influencia del grado de transparencia semántica y del grado de afijación}

Si se considera que las informaciones morfológicas intervienen en el curso del reconocimiento de palabras morfológicamente complejas, tal como las investigaciones citadas en la sección anterior lo proponen, y que existen factores que intervienen durante el reconocimiento de las palabras habladas polimorfémicas, nos preguntamos en este trabajo si específicamente el grado de afijación y el grado de transparencia semántica son factores que determinan el tipo de proceso que sostendría el reconocimiento auditivo de las palabras morfológicamente complejas.

\subsection{Influencia del grado de transparencia semántica}

A partir de los resultados obtenidos en un experimento que utilizaba el «priming ${ }^{6}$ morfológico», Marslen-Wilson et al. (1994) mostraron que, en inglés, el grado de transparencia semántica influye sobre el reconocimiento de las palabras polimorfémicas.

También Wurm (1997), basándose en resultados obtenidos mediante una tarea de decisión léxica, concluyó que la transparencia semántica tiene un efecto facilitador durante el reconocimiento de palabras inglesas complejas.

Apoyándonos en esta base teórica y experimental, nos preguntamos si el grado de transparencia semántica influye también en el reconocimiento de palabras habladas polimorfémicas en francés.

\subsection{Influencia del grado de afijación —-prefijación y sufijación}

Con la ayuda de una tarea de decisión léxica, Wurm (1997) observó que el grado de prefijación influía sobre los tiempos de reacción utilizados por los sujetos para reconocer las palabras polimorfémicas del inglés. Mostró que, cuanto más prefijada consideren los oyentes una palabra, más rápido se efectuará su reconocimiento. Con base en estos resultados, nos planteamos también si el grado de prefijación, y añadimos el de sufijación, tienen efecto durante el reconocimiento auditivo de las palabras complejas en los sujetos francófonos o si el efecto se presenta sólo en lengua inglesa.

\section{Diferencia de tratamiento para las palabras prefijadas y para las sufijadas}

En un experimento de decisión léxica, Colé et al. (1986) encontraron una diferencia de tratamiento entre las palabras prefijadas y las sufijadas. Obtuvieron un efecto de la estructura morfológica en el tratamiento de las palabras sufijadas. Contrariamente, este efecto no se observó para las palabras prefijadas. 
Beauvillain (1996) también evaluó la asimetría encontrada por Colé y colaboradores entre las palabras prefijadas y las sufijadas. Esta autora concluye que sí existe descomposición morfológica para los dos tipos de palabras afijadas, según la organización secuencial de los constituyentes morfémicos en el interior de una palabra. Para las palabras sufijadas, afirma que la raíz se integra primero. Esta integración tiene como efecto la activación de todas las palabras sufijadas ligadas a esta raíz.

Para las palabras prefijadas, propone que, según el punto de vista de un proceso de acceso de izquierda a derecha, el reconocimiento de las palabras prefijadas comienza en el punto de unicidad ${ }^{7}$ de las palabras implicadas. Por consiguiente, la cantidad de candidatas, en el caso de palabras prefijadas, no debería estar determinada por relaciones morfológicas.

Finalmente, Meunier (1997) encontró en sus experimentos un efecto de frecuencia de superficie $^{8}$ durante el tratamiento de las palabras prefijadas. Ella propone que no hay descomposición morfológica durante su tratamiento (raíz + prefijo). En cuanto a las sufijadas, halló un efecto de frecuencia de superficie y de frecuencia acumulada ${ }^{9}$, por lo que propuso que sí existe descomposición morfológica (raíz + sufijo) para este tipo de palabras. Así, postula la existencia de una asimetría en los procedimientos de tratamiento para las palabras prefijadas y para las sufijadas.

\section{Hipótesis}

Después de haber presentado una revisión de los experimentos más decisivos relacionados con los grados de afijación y de transparencia semántica, planteamos las siguientes hipótesis:

\section{Efecto de la morfología sobre el tratamiento de las palabras complejas}

Al respecto, esperamos que:

1. La estructura de las palabras intervenga durante el tratamiento de las palabras complejas, tal como los modelos descomposicionales y mixtos lo proponen (véase Arrieta 2002). Esperamos entonces una diferencia en los tiempos de reacción de las palabras categorizadas previamente como afijadas y no afijadas.

Operacionalmente, se espera la situación siguiente:

1.1. Los tiempos de reacción de las palabras afijadas serán mayores o menores a los tiempos de reacción de las palabras no afijadas.

\section{Efecto de la transparencia semántica sobre el tratamiento de las palabras complejas}

\section{Esperamos que:}

2. El grado de transparencia semántica determine el tipo de proceso que sostendría el reconocimiento: cuanto más transparente se considere una palabra en el plano semántico, es más probable que se aplique un análisis morfológico durante su tratamiento. 
Operacionalmente, esta hipótesis implicaría que:

2.1. Si el grado de transparencia semántica facilita el reconocimiento de las palabras habladas polimorfémicas en francés, las palabras juzgadas como transparentes en el plano semántico serán reconocidas más rápidamente que las palabras juzgadas opacas sobre el plano semántico. Se espera una diferencia entre los tiempos de reacción de las palabras juzgadas como transparentes y las juzgadas como opacas en el plano semántico: el tiempo de reacción de las palabras transparentes será menor al de las palabras opacas.

\section{Efecto del grado de afijación}

Esperamos que:

3. El grado de afijación influencie el tipo de proceso que sostendría el reconocimiento: cuanto más afijada se considere una palabra, más probabilidades habrá de que se aplique un análisis morfológico durante su tratamiento.

Operacionalmente,

3.1. Si el grado de afijación influye sobre el reconocimiento de palabras morfológicamente complejas, se espera que las palabras juzgadas como «más afijadas» sean reconocidas más rápidamente que las «poco afijadas» (las pseudoafijadas y las monomorfémicas). Esto se traducirá en una diferencia entre los tiempos de reacción de las palabras «afijadas» y los tiempos de reacción de las «poco afijadas»: los tiempos de reacción de las palabras afijadas serán menores que los de las palabras «poco afijadas».

Diferencia de tratamiento para las palabras prefijadas y para las palabras sufijadas

En nuestra investigación,

4. Si el tipo de afijo interviene durante el reconocimiento, esperamos una diferencia entre las palabras consideradas como prefijadas y aquellas consideradas como sufijadas, tal como Colé et al. (1986) y Meunier (1997) lo mostraron. Suponemos entonces que, tal como lo proponen las autoras, sólo habrá un efecto de la morfología para las palabras sufijadas.

Operacionalmente, la hipótesis precedente será corroborada por los siguientes hechos:

4.1. Una diferencia en los tiempos de reacción de las palabras categorizadas como afijadas (complejas) y entre los de las palabras categorizadas como no afijadas (monomorfémicas).

4.2. Una diferencia en el tiempo de reacción de las palabras categorizadas como prefijadas y las categorizadas como sufijadas. 
4.3. Un efecto de frecuencia de superficie que va a diferenciar a las palabras prefijadas y a las palabras sufijadas.

4.4. Una correlación entre los tiempos de reacción de las palabras afijadas y las variables morfológicas (transparencia semántica y grado de afijación). Según nuestra hipótesis, esta correlación sería mayor para las palabras sufijadas.

\section{Sujetos}

Con el fin de cumplir con los requisitos de un curso de Psicolingüística de primero o de segundo ciclo (bachillerato), 25 alumnos de la Facultad de Psicología de la Universidad de Ginebra participan en el experimento de decisión léxica. Todos son hablantes nativos del francés (han hablado únicamente esta lengua hasta la edad de 5 años).

\section{Material experimental}

El material experimental utilizado en esta tarea de decisión léxica está constituido por las mismas 40 tríadas de palabras recopiladas para los cuestionarios $(n=120)$ (véase Arrieta 2003). Cada tríada está constituida ya sea por una palabra prefijada, una pseudoafijada y una palabra monomorfémica de control o por una palabra sufijada, una pseudoafijada y una monomorfémica de control. Además, en el interior de cada tríada, se controló la frecuencia de uso en la lengua, la longitud (expresada en el número de fonemas) y la categoría gramatical.

Se construyeron dos listas. Cada lista contenía 60 palabras experimentales (20 palabras afijadas, 20 pseudoafijadas y 20 palabras de control, las monomorfémicas), así como 60 no palabras ${ }^{10}$, construidas sustituyendo uno o varios fonemas de las palabras seleccionadas.

Por ejemplo, la palabra ululer generó la no palabra udufer. Nos aseguramos de que las no palabras siguieran las restricciones fonológicas del francés pasando las listas de palabras y las de las no palabras a dos jueces, para verificar que las palabras de origen no se pudieran deducir de la forma fonológica de las no palabras; sólo en 5 casos la relación entre las palabras de origen y las no palabras asociadas se descubrió, por lo que el material escogido se consideró adecuado para realizar el experimento.

\section{Procedimiento}

Como se señaló antes, se utiliza en este trabajo la tarea de decisión léxica. Para esta tarea, los sujetos, individualmente, están equipados con un casco estéreo, en una cabina insonorizada. Escuchan en una misma sesión de aproximadamente 20 minutos la lista de 240 estímulos. Antes de comenzar el experimento, cada sujeto coloca sus manos de manera que pueda responder lo más rápidamente posible: los índices de cada mano deben estar colocados sobre los botones. Una vez que la grabación con la lectura de la lista comienza, el sujeto tiene por tarea apoyar lo más rápidamente posible, con su mano dominante, el botón verde si considera que el ítem escuchado es una palabra o el botón rojo si considera que el ítem escuchado es una no palabra. 
Simultáneamente, la computadora graba únicamente los tiempos de respuesta asociados a las palabras experimentales. Cada sujeto escucha una de las dos listas (A o B) y trata todas las palabras experimentales. Se construyó, con los 240 estímulos, un bloque 1 y un bloque 2. La lista A contiene primero los estímulos del bloque 1 y luego los del bloque 2. Contrariamente, la lista B presenta primero los estímulos del bloque 2 y luego los estímulos del bloque 1. El interés de tener dos listas es controlar que los efectos observados no puedan ser atribuidos al lugar de los ítemes de las listas. Antes del desarrollo de la tarea de decisión léxica, los sujetos escuchan un entrenamiento, para verificar la calidad del sonido, la claridad de las consignas y la conexión del equipo de laboratorio. Una vez que el sujeto manifiesta su aprobación y si todo está en orden, el experimento de decisión léxica comienza.

\section{Plan experimental}

En la investigación se controlarán las siguientes variables:

\section{Variables dependientes}

El tiempo de reacción y el número de errores.

\section{Variables controladas}

La frecuencia de superficie de las palabras y la longitud expresada en número de sílabas (fonemas).

\section{Variables independientes}

La estructura de las palabras y de las no palabras según las condiciones experimentales ejemplificadas en el cuadro 1.

Cuadro 1

Condiciones experimentales

\begin{tabular}{lllccc}
\hline Condición & Tipo morfológico & Ejemplo & Frecuencia & Lg. & P.U. \\
\hline \multirow{2}{*}{ Palabras simples } & monomorfémicas & sésame & 42 & 5 & 5 \\
& pseudoprefijadas & décréter & 7 & 7 & 6 \\
& pseudosufijadas & pactole & 12 & 6 & 5 \\
\hline \multirow{2}{*}{ Palabras complejas } & prefijadas & décompter & 46 & 6 & 6 \\
& sufijadas & pactiser & 123 & 7 & 5 \\
\hline
\end{tabular}

Covariables : el grado de transparencia semántica y el grado de prefijación.

\section{Resultados de la decisión léxica}

Como ningún sujeto cometió más de 14 errores, no descartamos a ningún sujeto de los análisis estadísticos. No obstante, cuatro palabras no fueron reconocidas como tales por la mitad 
de los sujetos: centaure $(n=15)$, élider $(n=14)$, erratique $(n=15)$ y gavotte $(n=14)$. Por lo tanto, las palabras que forman parte de esas tríadas (respectivamente, ramage, rameur; aliéner, aligner; y indigène, indistinct; gavage y sésame) no fueron consideradas en el análisis estadístico.

Cuadro 2

Tiempos de reacción de la decisión léxica

Tiempo de reacción

(inicio de palabra)
Tiempo de reacción

(final de palabra)

\section{Afijadas \\ prefijadas \\ sufijadas}

Pseudoafijadas

pseudoprefijadas

pseudosufijadas

Monomorfémicas

asoc. a prefijadas

asoc. a sufijadas

\begin{abstract}
$903.22 \mathrm{~ms}$
\end{abstract}
$901.577 \mathrm{~ms}$

$863.22 \mathrm{~ms}$

$915.708 \mathrm{~ms}$

$878.951 \mathrm{~ms}$

$915.708 \mathrm{~ms}$
$237.1 \mathrm{~ms}$

$202.4 \mathrm{~ms}$

$215.9 \mathrm{~ms}$

$268.6 \mathrm{~ms}$

$273.3 \mathrm{~ms}$

$249.5 \mathrm{~ms}$

Influencia de la estructura de las palabras morfológicamente complejas en reconocimiento auditivo: efecto principal de la naturaleza morfológica de las palabras percibidas

Para evidenciar un efecto de estructura morfológica de palabras, comparamos los datos obtenidos para las palabras pseudoafijadas con los obtenidos para las palabras no afijadas - en forma similar a lo que Taft (1981) propone-; y, por otro lado, los de las palabras monomorfémicas no analizables en el plan morfológico — como lo realizó Pillon (1998).

Como las palabras prefijadas podrían ser procesadas de manera diferente a las palabras sufijadas, distinguimos los análisis a partir de estas dos categorías de palabras.

\section{Efecto de la pseudo-prefijación}

El efecto de la pseudoprefijación / sufijación puede ser calculado de dos maneras:

a) Comparando los datos obtenidos para las palabras prefijadas/sufijadas con los obtenidos para las palabras pseudosufijadas que contienen un segmento formalmente similar al prefijo de las palabras prefijadas.

b) Comparando los datos obtenidos para las palabras pseudoprefijadas/sufijadas con los obtenidos para las palabras monomorfémicas similares (de igual frecuencia, longitud...).

\section{Medida de tiempo de reacción a partir del inicio de las palabras}

En la literatura, los tiempos de reacción se miden desde el inicio de la palabra para determinar si estos se producen antes del final del estímulo. Lo anterior se evidenciaría cuando los 
tiempos de reacción son muy breves. Sin embargo, si existe una diferencia entre los tiempos de reacción, habría que preguntarse si esta se debe a una diferencia de tratamiento de los estímulos o a su duración física. Así, en esta investigación, para centrarnos en la diferencia debida al tratamiento de las palabras, también se han medido los tiempos de reacción desde el final de las palabras, para eliminar la posibilidad de explicar las diferencias obtenidas por un efecto de la duración física de las palabras.

\section{Medida de tiempos de reacción a partir del inicio de las palabras}

La diferencia entre las prefijadas y las pseudoprefijadas (cf. cuadro1) no es significativa más que en el análisis sujeto por sujeto $[\mathrm{F} 1(1,24)=23.975, \mathrm{p}<0.0001]$; $[\mathrm{F} 2(1,34)=$ $2.179, \mathrm{p}=0.1491]$. Si la mayoría de sujetos reconocen en general más rápidamente las pseudoprefijadas, no se puede, desgraciadamente, generalizar este resultado a todas las parejas de palabras seleccionadas.

No se nota una diferencia significativa entre las palabras monomorfémicas y las pseudoprefijadas (cf. cuadro 2), ya sea en el análisis sujeto por sujeto o ítem por ítem $[\mathrm{F} 1(1,24)=$ 2.194, $\mathrm{p}=0.1515]$; [F2 (1,34), $\mathrm{p}<1$ ]. Reconocer un pseudoprefijo no necesita más tiempo que reconocer una palabra monomorfémica.

\section{Medida de los tiempos de reacción a partir del final de las palabras}

La diferencia entre las prefijadas y las pseudoprefijadas (cf. cuadro 1) no es significativa más que en el análisis sujeto por sujeto [F1 $(1,24)=10.994, \mathrm{p}=0.0029]$; [F2 $(1,34)<1]$. De nuevo, si la mayoría de los sujetos reconocen más rápidamente las pseudoprefijadas que las prefijadas, no se puede generalizar el resultado a todos los pares de palabras seleccionadas.

Se nota, contrariamente, una diferencia significativa entre las palabras monomorfémicas y las pseudoprefijadas (cf. cuadro 2$)[\mathrm{F} 1(1,24)=30.933, \mathrm{p}<0.0001]$; $[\mathrm{F} 2(1,34)=6.697$, $\mathrm{p}=0.0141]$. Esta vez, las palabras pseudoprefijadas se reconocen más rápidamente que las palabras monomorfémicas.

\section{Efecto de pseudosufijación}

Medida de tiempos de reacción a partir del principio de las palabras

La comparación de los datos obtenidos para las palabras sufijadas y pseudosufijadas (cf. cuadro 3) no alcanzó el nivel de significatividad más que en el análisis sujeto por sujeto $[\mathrm{F} 1(1,24)=2.694, \mathrm{p}=0.1138] ;[\mathrm{F} 2<1]$. Si la mayoría de sujetos reconoce más rápidamente las sufijadas que las pseudosufijadas, no se puede generalizar el resultado a todos los pares de palabras seleccionadas.

Si se comparan los datos obtenidos para las palabras pseudosufijadas y para las monomorfémicas (cf. cuadro 3), ningún efecto significativo aparece $[\mathrm{F} 1(1,24)<1]$; [F2 $(1,34)$ $<1]$. Las palabras monomorfémicas no toman más tiempo para ser reconocidas que las palabras pseudosufijadas. 
Medida de los tiempos de reacción a partir del final de palabra

La comparación de los datos obtenidos para las palabras sufijadas y pseudosufijadas (cf. cuadro 3) alcanza el nivel de significatividad para el análisis sujeto por sujeto y es tendencioso para el análisis ítem por ítem [F1 $(1,24)=63.047, \mathrm{p}<0.0001]$; [F2 $(1,34)=$ 2.943, $\mathrm{p}=0.0954]$. Las pseudosufijadas parecen ser reconocidas más lentamente que las palabras sufijadas.

Si se comparan los datos obtenidos para las pseudosufijadas y para las palabras monomorfémicas (cf. cuadro 3), observamos un efecto significativo, pero únicamente en el análisis sujeto por sujeto $[\mathrm{F} 1(1,24)=4265, \mathrm{p}=0.0499]$; $[\mathrm{F} 2(1,34)<1]$. Si los sujetos reconocen más rápidamente las palabras monomorfémicas asociadas a las sufijadas que las pseudosufijadas, no se puede generalizar este resultado a todas las parejas de palabras seleccionadas.

Teniendo en cuenta la ausencia de efectos cuando los tiempos de reacción se calculan a partir del inicio de las palabras, los análisis posteriores sólo contemplarán los datos obtenidos a partir de los tiempos de reacción medidos desde el final de la palabra.

\section{Efecto de la frecuencia de superficie}

Evaluamos la relación entre los tiempos de reacción para cada ítem con su frecuencia respectiva: «cuanto más frecuente es un ítem, ¿es reconocido más rápidamente?».

Para responder a esta pregunta, calculamos un índice de correlación entre los tiempos de reacción (descontados a partir del final de las palabras) y la frecuencia determinada en el Brulex (Content et al. 1990). Para el grupo de palabras prefijadas, el índice se eleva a -0.389 (dl 1, p= 0.004). Para el grupo de las sufijadas, se eleva a -0.163 (dl. 1, p>1). Si la frecuencia de superficie de las palabras influye realmente en el perfil de resultados para las palabras prefijadas — cuanto más frecuente es una palabra, más rápidamente es reconocida—, no influye significativamente sobre los datos obtenidos para las palabras sufijadas.

Si se comparan estos resultados con los obtenidos por Colé et al. (1986) y Meunier (1997), se puede decir que, en nuestro trabajo, se puede también hablar de una diferencia entre las palabras prefijadas y las sufijadas.

En nuestros experimentos, controlamos solamente la frecuencia de superficie y no la frecuencia acumulada. Sin embargo, una diferencia entre las palabras prefijadas y las palabras sufijadas se constató: hay un efecto de la frecuencia de superficie solamente para las palabras prefijadas. Este efecto está ausente para las palabras sufijadas.

\section{Efecto del grado de prefijación y de sufijación}

a) Según los datos provenientes de los cuestionarios auditivos

Con el fin de evaluar la relación entre el grado de prefijación/sufijación de las palabras y el tiempo necesario para su reconocimiento, construimos dos análisis de correlaciones. Para el grupo de prefijadas, no aparece ningún vínculo $(r=-0.135, d l=1, p=0.3331)$. Contrariamente, la correlación para las palabras sufijadas es significativa $(\mathrm{r}=-0.342, \mathrm{dl}=1, \mathrm{p}=0.011)$. Así, no es más que para las palabras sufijadas que la presencia de un afijo influencia los procedimientos de reconocimiento auditivo. 
b) Según los datos provenientes de los cuestionarios escritos

Para el grupo de las palabras prefijadas, no se presentó ningún vínculo significativo $(\mathrm{r}=-0.098$, dl.1, p mayor a 0.1), contrariamente al grupo de sufijadas $(\mathrm{r}=-0.316$, dl. 1 , $\mathrm{p}=0.0195$ ). Sólo para las palabras sufijadas la presencia de un afijo influencia los procedimientos de reconocimiento auditivo.

\section{Efecto del grado de transparencia semántica}

Con el objetivo de evaluar la relación entre el grado de transparencia semántica de las palabras y el tiempo necesario para su reconocimiento, realizamos análisis correlacionales utilizando los resultados de la decisión léxica y los datos provenientes de los cuestionarios auditivos y escritos.

a) Según los datos de los cuestionarios auditivos

Para el grupo de las palabras prefijadas, la correlación no es muy significativa $(\mathrm{r}=-0.235, \mathrm{dl}=1, \mathrm{p}=0.0873)$. Contrariamente, para el grupo de las palabras sufijadas, la correlación alcanzó el nivel de significación $(\mathrm{r}=-0.318, \mathrm{dl}=1, \mathrm{p}=0.0178)$. Así, no sería más que para las palabras sufijadas que los elementos que componen estas palabras intervienen durante los procedimientos de reconocimiento auditivo.

b) Según los datos provenientes de los cuestionarios escritos

Solamente para el grupo de sufijadas, la correlación alcanzó el nivel de significatividad (para el grupo de prefijadas: $\mathrm{r}=-0.174$, dl. $1, \mathrm{p}>0.1$ y para el grupo de las sufijadas: $\mathrm{r}=-0.348, \mathrm{dl} .1, \mathrm{p}=0.0094)$. Por lo tanto, solo para las palabras sufijadas los elementos que las componen intervienen durante los procedimientos de reconocimiento auditivo.

\section{Cuadro 3}

Resumen de resultados de la decisión léxica (a partir del final de las palabras)

\begin{tabular}{lll}
\hline Efectos evaluados & Grupo de prefijadas & Grupo de sufijadas \\
\hline Efecto de pseudoafijación & & \\
Diferencia pseudo-afijadas - & $\mathrm{F} 1(1,24)=10.994, \mathrm{p}=0.0029$ & $\mathrm{~F} 1(1,24)=63.047, \mathrm{p}<0.0001$ \\
afijadas & $\mathrm{F} 2(1,34)<1$ & $\mathrm{~F} 2(1,34)=2.943, \mathrm{p}=0.0954$ \\
$\begin{array}{l}\text { Diferencia pseudo-afijadas- } \\
\text { monomorfémicas }\end{array}$ & $\mathrm{F} 1(1,24)=30.933, \mathrm{p}=0.0001$ & $\mathrm{~F} 1(1,24)=4.265, \mathrm{p}=0.0499$ \\
\hline $\begin{array}{l}\text { Efecto de frecuencia } \\
\text { de superficie }\end{array}$ & $\mathrm{F} 2(1,34)=6.697, \mathrm{p}=0.0141$ & $\mathrm{~F} 2(1,34)<1$ \\
\hline
\end{tabular}


Cuadro 3

Resumen de resultados de la decisión léxica (a partir del final de las palabras)

\begin{tabular}{|c|c|c|}
\hline Efectos evaluados & Grupo de prefijadas & Grupo de sufijadas \\
\hline \multicolumn{3}{|l|}{ Efecto de pseudoafijación } \\
\hline $\begin{array}{l}\text { Diferencia pseudo-afijadas- } \\
\text { afijadas } \\
\text { Diferencia pseudo-afijadas- } \\
\text { monomorfémicas }\end{array}$ & $\begin{array}{l}F 1(1,24)=10.994, p=0.0029 \\
F 2(1,34)<1 \\
F 1(1,24)=30.933, p=0.0001 \\
F 2(1,34)=6.697, p=0.0141\end{array}$ & $\begin{array}{l}F 1(1,24)=63.047, p<0.0001 \\
F 2(1,34)=2.943, p=0.0954 \\
F 1(1,24)=4.265, p=0.0499 \\
F 2(1,34)<1\end{array}$ \\
\hline $\begin{array}{l}\text { Efecto de frecuencia } \\
\text { de superficie }\end{array}$ & $\mathrm{r}=-0.389, \mathrm{dl} .1, \mathrm{p}=0.004$ & $\mathrm{r}=-0.163, \mathrm{dl} .1, \mathrm{p} 0.1$ \\
\hline Efecto del grado de afijación & $\begin{array}{l}\mathrm{r}=-0.135, \mathrm{~d} l .1, \mathrm{p}=0.3331 \\
\text { (modalidad auditiva) } \\
\mathrm{r}=-0.098, \mathrm{dl} .1, \mathrm{p}>0.1 \\
\text { (modalidad visual) }\end{array}$ & $\begin{array}{l}\mathrm{r}=-0342, \mathrm{dl} .1, \mathrm{p}=0.011 \\
\text { (modalidad auditiva) } \\
\mathrm{r}=-0.316, \mathrm{dl} .1, \mathrm{p}=0.0195 \\
\text { (modalidad visual) }\end{array}$ \\
\hline $\begin{array}{l}\text { Efecto del grado de } \\
\text { transparencia semántica }\end{array}$ & $\begin{array}{l}\mathrm{r}=-0.235, \mathrm{dl} .1, \mathrm{p}=0.0873 \\
\text { (modalidad auditiva) } \\
\mathrm{r}=-0.174, \mathrm{dl} .1, \mathrm{p}>0.1 \\
\text { (modalidad visual) }\end{array}$ & $\begin{array}{l}\mathrm{r}=-0318, \mathrm{dl} .1, \mathrm{p}=0.0187 \\
\text { (modalidad auditiva) } \\
\mathrm{r}=-0.348, \mathrm{dl} .1, \mathrm{p}=0.0094 \\
\text { (modalidad visual) }\end{array}$ \\
\hline
\end{tabular}

\section{Discusión de resultados}

Con la utilización de una tarea de decisión léxica, nos propusimos evaluar si las características de los ítemes tienen implicaciones sobre los tiempos de reacción de los sujetos y evaluar si hay relaciones entre la velocidad a la cual las palabras morfológicamente complejas son tratadas y sus grados de transparencia semántica y de afijación.

Así, a partir de los resultados, obtuvimos la confirmación para algunas de nuestras hipótesis. Por una parte, obtuvimos tiempos de respuesta diferentes para las palabras afijadas y para las no afijadas (cf. cuadro 3), es decir, que nuestra hipótesis relativa al efecto de la estructura de las palabras y a su tratamiento se ha confirmado.

En lo que respecta al grado de afijación, nuestras hipótesis se han confirmado parcialmente. A partir de nuestros resultados, la relación entre los tiempos de reacción y el grado de afijación ha sido representativa solamente para las palabras sufijadas. Solo para estas últimas la presencia de un afijo influencia los procedimientos de reconocimiento.

Las hipótesis relativas al grado de transparencia semántica también se han confirmado parcialmente. De nuevo, según nuestros resultados, se ha obtenido un efecto de transparencia semántica solamente para las palabras sufijadas. Solo para este tipo de palabras los elementos que las componen intervendrían durante los procedimientos de reconocimiento auditivo.

Para la diferencia de tratamiento entre las palabras prefijadas y las palabras sufijadas, confirmamos nuestras hipótesis: constatamos una asimetría entre estos dos tipos de palabras. Por un lado, encontramos que los tiempos de reacción para las palabras afijadas eran más extensos que los de las palabras no afijadas. Además, encontramos un efecto de frecuencia de superficie solamente para las palabras prefijadas y no para las palabras sufijadas. Y, finalmente, 
una correlación entre los tiempos de reacción de las palabras sufijadas y las variables morfológicas (transparencia semántica y grado de afijación). La asimetría es clara: estas correlaciones son significativas únicamente para las palabras sufijadas.

\section{Conclusiones}

A lo largo de este trabajo, hemos presentado algunos resultados empíricos que han generado polémica, en el campo del reconocimiento de palabras polimorfémicas, en lo relativo a la influencia de la estructura morfológica de las palabras durante su tratamiento y, principalmente, a propósito de las variables que pondrían en evidencia la influencia de la morfología.

Centramos nuestro interés en dos factores estudiados en lengua inglesa (Marslen-Wilson 1994 y Wurm 1997): el efecto del grado de transparencia semántica y el efecto del grado de afijación.

Evaluamos los efectos de las variables mencionadas, así como los posibles efectos de su interacción, durante el tratamiento de las palabras habladas prefijadas y sufijadas. El objetivo de este estudio era obtener datos, en lengua francesa, sobre el efecto de estas dos variables, dado que los resultados existentes sólo se han comprobado para el inglés.

Tal como lo presentamos en la sección de resultados, obtuvimos, a partir de la tarea de decisión léxica, los efectos siguientes:

un efecto de pseudoafijación,

un efecto de frecuencia,

un efecto del grado de afijación,

y un efecto del grado de transparencia semántica.

Estos resultados, en el contexto de las premisas teóricas de los modelos de reconocimiento de las palabras complejas (ver Arrieta 2002: 215-29) siguen la línea no homogénea presentada por los resultados empíricos de las investigaciones morfológicas.

Por un lado, nuestros resultados son incompatibles con las premisas de la hipótesis del listado exhaustivo. Este modelo no hace ninguna interpretación de los efectos obtenidos en los resultados. Al contrario, según este modelo, las informaciones morfológicas no intervienen durante el reconocimiento de palabras habladas complejas y, por consiguiente, debería haber una ausencia de efectos de las variables descomposicionales mencionadas anteriormente.

Sin embargo, los efectos encontrados sugieren la influencia de la información morfológica durante el tratamiento de las palabras complejas polimorfémicas. Así, nuestros datos son más compatibles con las proposiciones de los modelos descomposicionales y con las de los modelos mixtos, que sí toman en cuenta estas variables.

\section{Efecto de pseudoafijación}

El efecto de seudoafijación ha sido utilizado, en las investigaciones psicolingüísticas, como indicador de una evidencia de descomposición morfológica. En lo que se refiere a este efecto, encontramos una diferencia entre las palabras prefijadas y las palabras sufijadas. 
Para el grupo de palabras prefijadas, obtuvimos que las palabras pseudoprefijadas eran reconocidas más rápidamente que las palabras prefijadas. Este resultado podría indicar un tratamiento más rápido de las palabras pseudoprefijadas que de las palabras prefijadas.

Como ya señalamos, este resultado no es compatible con la hipótesis del listado exhaustivo, que predice un tratamiento idéntico para los dos tipos de palabras en cuestión.

El modelo descomposicional predice un tratamiento diferente para estos dos tipos de palabras: un tratamiento más largo para las palabras pseudoprefijadas y un tratamiento más corto para las palabras prefijadas. El modelo predice que los tiempos de reacción asociados a las palabras pseudoafijadas deberían aumentar con respecto a las palabras prefijadas. Este retraso sería explicable por el fracaso de la búsqueda efectuada después del proceso de descomposición (supresión obligatoria del afijo). En nuestros resultados, el efecto de pseudoprefijación no ha sido obtenido en la dirección del modelo de descomposición.

Contrariamente a las predicciones del modelo, en nuestro trabajo las palabras prefijadas se identifican más lentamente que las palabras pseudoafijadas. Sin embargo, nuestros resultados sugieren, como el modelo descomposicional lo predice, una influencia significativa de la morfología. Para el grupo de las palabras sufijadas, nuestros resultados son compatibles con las proposiciones del modelo descomposicional: las palabras pseudosufijadas son asociadas a tiempos de reacción más largos que los de las palabras sufijadas. Situación explicable, según el modelo, por el tiempo adicional tomado por el proceso de tratamiento debido al fracaso de la primera búsqueda (para más detalles del modelo, véase Arrieta 2002: 215-29).

Estos resultados sugieren, como los resultados obtenidos para las palabras prefijadas, que la morfología interviene durante el tratamiento de las palabras habladas polimorfémicas.

En el contexto de los modelos mixtos, este efecto de la pseudoafijación no es específicamente interpretable. Por un lado, para el modelo AAM (Augmented Addressed Morphology Model), no habría costos de tratamiento para el acceso léxico de las palabras pseudoafijadas. Estas palabras son consideradas como palabras monomorfémicas y van a ser tratadas como cualquier otra palabra simple o monomorfémica.

Para el Race Model, no hay predicciones precisas para el tratamiento de las palabras pseudoafijadas. El modelo señala que estas palabras serían tratadas por la vía directa, dado que se trata de palabras monomorfémicas.

El Meta Model es un poco más específico en lo que concierne a la existencia de efectos de la pseudoafijación. Señala que el costo de tratamiento debido a la pseudoafijación sería resuelto durante la etapa de retroalimentación, mientras que se realiza el cálculo del sentido para las palabras complejas. Sin embargo, el modelo no ofrece predicciones más precisas a propósito de este efecto y nuestros resultados no pueden ser interpretados según esta posición.

\section{Efecto de frecuencia}

Nuestros resultados han puesto también en evidencia un efecto de la frecuencia de superficie. Una vez más, este efecto se encontró solamente para un tipo de palabras: las palabras sufijadas.

Para el modelo del listado exhaustivo, el efecto de frecuencia no es un indicio de influencia de la morfología, dado que no tiene en cuenta las variables descomposicionales.

Contrariamente, en el caso del modelo descomposicional, el efecto de la frecuencia ha servido como indicio de una evidencia de descomposición morfológica durante el tratamiento de las palabras habladas polimorfémicas. 
Nuestros resultados muestran un efecto de la frecuencia de superficie para las palabras prefijadas, pero ningún efecto de este tipo de frecuencia para las palabras sufijadas. Estos resultados no van totalmente en la misma dirección de los encontrados por Colé (1986) y Meunier (1997), pero evidencian la asimetría reportada entre las palabras prefijadas y las palabras sufijadas. Estas autoras encontraron un efecto de la frecuencia de superficie para los dos tipos de palabras complejas, mientras que la diferencia entre estos dos tipos de palabras se evidenció por un efecto de frecuencia acumulada obtenida solamente para las palabras sufijadas.

En nuestra investigación, la frecuencia acumulada no se controló. Por esta razón, no podemos discutir sobre un eventual efecto de este tipo de frecuencia ni interpretar totalmente nuestros resultados según las posiciones de las autoras mencionadas. A pesar de esto, nuestros resultados evidencian una asimetría entre las palabras prefijadas y las palabras sufijadas.

\section{Efecto de la transparencia semántica}

Ya señalamos que el efecto de la transparencia semántica no ha sido tomado en cuenta más que por los modelos mixtos. Ni los modelos del listado exhaustivo ni los modelos descomposicionales hacen predicciones relativas a este efecto. Por esta razón, nuestros resultados serán interpretados a partir de las predicciones de los modelos mixtos.

Dichos modelos predicen que la transparencia semántica es una de las propiedades léxicas que va a determinar las condiciones por las cuales el acceso léxico se realiza por una vía o por la otra. Para estos modelos, tal noción implica la naturaleza de la relación entre una raíz y su(s) afijo(s) (véase Arrieta 2002: 215-29).

En nuestra investigación, los datos indican de nuevo una diferencia entre las palabras prefijadas y las palabras sufijadas.

Obtuvimos un claro efecto de transparencia semántica para las palabras sufijadas; pero, para las palabras prefijadas, la correlación entre el tiempo de reacción y el grado de transparencia semántica no es muy significativa.

Así, nuestros resultados son compatibles, para las palabras sufijadas, con las hipótesis propuestas por los modelos mixtos que consideran la transparencia semántica como una de las propiedades importantes durante el tratamiento de las palabras morfológicamente complejas.

El modelo predice que la transparencia semántica estará implicada durante la etapa que ellos denominan retroalimentación. En el caso de nuestros resultados, el efecto de transparencia semántica estaría implicado durante el tratamiento de las palabras sufijadas (y transparentes). Durante la etapa de retroalimentación, la relación semánticamente transparente entre las palabras sufijadas (y transparentes) y sus constituyentes sería modelada como un «overlap» entre la serie de las representaciones semánticas de las palabras sufijadas y la serie de sus constituyentes.

Al respecto, el Race Model predice que las palabras morfológicamente complejas, semánticamente transparentes, tomarán menos tiempo para ser analizadas morfológicamente que las palabras que son menos transparentes a ese nivel.

Observamos que, en nuestra investigación, las palabras sufijadas (semánticamente transparentes) se reconocen más rápidamente que las palabras pseudosufijadas y monomorfémicas no transparentes.

Para las palabras prefijadas, esta predicción no se confirmó. Encontramos que las palabras prefijadas (y transparentes) no se reconocieron más rápidamente que las palabras pseudoafijadas ni que las monomorfémicas. 


\section{Efecto de grado de afijación}

En nuestro trabajo, obtuvimos una asimetría entre los datos para las palabras prefijadas y los de las palabras sufijadas. Hallamos un efecto del grado de afijación solamente para las palabras sufijadas. De nuevo, la relación entre los tiempos de reacción obtenidos en la tarea de decisión léxica y los índices de grado de afijación es significativa solamente para las palabras sufijadas.

Como el efecto de la transparencia semántica, el efecto del grado de afijación ha sido tomado en cuenta por lo modelos que consideran la influencia de los factores léxicos durante el tratamiento de las palabras complejas, es decir, los modelos de dos vías.

Por una parte, para el Race Model, sería muy importante determinar un verdadero afijo y sus características por las consecuencias que esto tendría en las relaciones raíz - afijo.

El modelo no predice en detalle un efecto de grado de afijación, pero sí menciona la importancia de determinar las características de un afijo por las implicaciones que esto tendría en el tratamiento de una forma compleja.

Por otra parte, para el Meta Model, el grado de afijación sería importante en la etapa de autorización propuesta para el tratamiento de palabras complejas.

Aunque las afirmaciones de estos dos modelos en lo que concierne el grado de afijación no son dados en detalle, y nuestros resultados no pueden ser totalmente interpretables según estas posiciones teóricas, sí podemos interpretarlos como indicadores de la influencia de la morfología durante el tratamiento de las palabras sufijadas.

\section{Las diferencias entre lenguas}

Nuestros resultados sobre el efecto de la transparencia semántica y sobre el grado de afijación han mostrado una diferencia entre las palabras prefijadas y las palabras sufijadas.

En el caso de las investigaciones en lengua inglesa, Marlen-Wilson (1994) y Wurm (1997) han mostrado un efecto facilitador de la variable transparencia semántica para el tratamiento de las palabras prefijadas y de las palabras sufijadas. Wurm (1997), por su parte, mostró el efecto facilitador del grado de afijación durante el reconocimiento de las palabras prefijadas en lengua inglesa.

Nuestros resultados han encontrado estos efectos, para la lengua francesa, únicamente para las palabras sufijadas. Nos parece que esta situación podría ser eventualmente explicable por las diferencias de organización morfológicas de las lenguas implicadas: el inglés y el francés, y, principalmente, por la posible interacción de otras variables (¿como la acentuación en inglés?) en la organización de las informaciones morfológicas concernientes a las palabras prefijadas.

Según nuestro criterio, la ausencia de efectos de transparencia semántica y del grado de afijación durante los resultados obtenidos para las palabras prefijadas podría dar lugar a otras investigaciones en las cuales las eventuales diferencias entre lenguas estén todavía más controladas. Es interesante resaltar el hecho de que, a pesar de que el inglés y el francés sean lenguas en las cuales la morfología no está tan alejada, las investigaciones realizadas sobre los factores morfológicos sí obtienen datos diferentes. ¿Cuál sería el caso de lenguas en las cuales la morfología está verdaderamente lejos de la del inglés? ¿Podrían las predicciones empíricas de los modelos, fundamentadas en datos obtenidos en lengua inglesa, verse enriquecidas por el estudio de otras lenguas? 


\section{Notas}

1. El grado de transparencia semántica es un criterio psicolingüístico y se refiere al hecho de que el significado de una palabra polimorfémica pueda deducirse del sentido de sus constituyentes, raíz y afijo.

2. El grado de afijación también es un criterio psicolingüístico. Se refiere al hecho de que una palabra afijada (prefijada o sufijada), pseudoafijada o no analizable morfológicamente sea juzgada por los hablantes nativos de una lengua particular como más, menos o nada afijada.

3. La realización de un análisis de tareas proporciona al psicólogo cognitivo una imagen de la secuencia lógica de pasos que conducen hacia una capacidad. Mediante el análisis de tareas es posible obtener insights de las teorías cognitivas existentes, de las formas como trabajan mentalmente los sujetos.

4. La tarea de decisión léxica pertenece a los procedimientos de medición del tiempo de reconocimiento léxico. En esta tarea, se le muestra al sujeto una secuencia de palabras (fonemas, letras...) para que éste la clasifique, con la mayor brevedad posible, como palabra o no-palabra (Belinchón 1992: 394).

5. El tiempo de reacción indica que cuanto mayor sea el tiempo transcurrido desde la presentación de un estímulo hasta la emisión de la respuesta por parte del sujeto, mayor número de procesos cognitivos están implicados.

6. El efecto de priming influye en el tiempo de reconocimiento de las palabras. Está relacionado con las condiciones de presentación de los estímulos. De esta manera, la presentación de ciertos estímulos léxicos con anterioridad a una palabra crítica puede facilitar o dificultar su reconocimiento, siempre y cuando tengan algún tipo de relación con ella. A estos estímulos se les denomina primes y al efecto que producen, priming (Belinchón 1992: 398).

7. Cada palabra posee «un punto óptimo de reconocimiento»o «punto de unicidad», momento a partir del cual llega una drástica reducción de los posibles candidatos a entrada léxica; es decir, el punto de unicidad hace referencia al punto en el que el candidato definitivo se destaca de los demás.

8. La frecuencia de superficie da cuenta de las veces que una palabra morfológicamente compleja aparece como una palabra completa específica.

9. La frecuencia acumulada da cuenta de las veces que aparece una raíz; asimismo, da cuenta de todas las formas afijadas que comparten esa misma raíz.

10. Las no palabras o logatomos consisten en secuencias de letras y sonidos que respeten las restricciones fonológicas y ortográficas de la lengua en estudio. Se emplean como parte del material experimental con el fin de detectar un «efecto de palabra vs. no palabra» en tareas de decisión léxica. Según experimentos psicolingüísticos, el tiempo que se tarda en aceptar cierto estímulo como palabra es inferior al invertido en rechazar estímulos categorizados como no-palabras, incluso si se comparan palabras infrecuentes con no-palabras.

\section{Bibliografía}

Arrieta, A. 2002. "Modelos psicolingüísticos de reconocimiento de palabras habladas polimorfémicas". Revista de Filología y Lingüística de la Universidad de Costa Rica. XXVIII (1): 215-29. 
2003. "Estudio de dos factores implicados en el reconocimiento de palabras: el grado de afijación y el grado de transparencia semántica". Revista de Filología y Lingüística de la Universidad de Costa Rica. XXIX (2): 131-146.

Belinchón, M. 1992. Psicología del lenguaje. Investigación y teoría. Madrid: Editorial Trotta, S.A.

Colé et al. 1989. "On the Representation and Processing of Prefixed and Suffixed Derived Words: a Differential Frequency Effect". Journal of Memory and Language. 28: 1-13.

Feldman, L.B. (Ed.). Morphological Aspects of Language Processing. Hillsdalen New Jersey: Erlbaum Associates Publishers.

Manelis, L. y D. Tharp. 1977. "The Processing of Affixed Words". Memory \& Cognition. 5: 690-5.

McQueen, J. y A. Cutler. 1998. "Morphology in Word Recognition”. En Spencer A. y Zwicky A.M. (Eds.): 406-27.

Meunier, F. 1997. Morphologie et Traitement du Langage Parlé. Thèse de Doctorat en Psychologie: Université René Descartes-Paris V.

Marslen-Wilson, W.D., X-L. Zhou, y M. Ford. 1996. "Morphology, modality and lexical architecture". En Yearbook of Morphology. Kluwer Academic Publishers: Netherlands.

Pillon, A. 1998. "The Pseudo-prefixation Effect in Visual Word Recognition: A True -Neither Strategic nor Orthographic-Morphemic Effect". The Quarterly Journal of Experimental Psychology. (1): 85-120.

Spencer A. y A.M. Zwicky (Eds.). The Handbook of Morphology. Oxford: Blackwell Publishers, 406-427.

Stolz, J.A. y L.B. Feldman. 1995. "The Role of Orthographic and Semantic Transparency of the base Morpheme in Morphological Processing". En L.B. Feldman (Ed.): 1009-129.

Taft, M. 1994. "Interactive-Activation as a Framework for Understanding Morphological Processing". Language and Cognitive Proceses. 9 (3): 271-94.

Taft, M. y K.I. Forster. 1975. "Lexical Storage and Retrieval of Prefixed Words". Journal of Verbal Learning and verbal Behavior. 14: 638-47.

Taft, M., G. Hambly y S. Kinoshita. 1986. "Visual and Auditory Recognition of Prefixed Words". Quaterly Journal of Experimental Psychology. 38 A: 351-66.

Tyler, L. y W.D. Marslen-Wilson. 1986. "The Effects of Context on the Recognition of Polimorphemic Words". Journal of Memory and Language. 25: 741-52. 
Tyler, L. et al. 1988. "Continuous and Discontinuous Acces in Spoken Word Recognition: The Role of Derivational Prefixes". Journal of Memory and Language: 27: 368-81.

Wurm Lee H. 1997. “Auditory Processing of Prefixed English Words is Both Continuous and Decompositional”. Journal of Memory and Language. 37: 438-61. 